\title{
Image Segmentation using Discontinuity-Based Approach
}

\author{
Rajiv Kumar \\ Bharathiar University, \\ Coimbatore (Tamil Nadu)
}

\author{
M.Arthanari \\ Bharathidasan School of \\ Computer Applications, \\ Erode (Tamil Nadu)
}

\author{
M.Sivakumar \\ Anna University, Coimbatore \\ (Tamil Nadu)
}

\begin{abstract}
Image segmentation is the process by which we segment a given image into several parts so that we can further analyzed each of these parts present in the image. We can extract some information by analyzing them and this information is useful for high-level machine vision application. There are numerous techniques of image segmentation available in literature. In this paper, we will analyze the discontinuity-based approach for image segmentation. The discontinuity-based segmentation can be classified into three approaches: point detection, line detection, and edge detection. In this paper, we will implement and analyses the result of these various approaches in MATLAB using Image Processing Toolbox (IPT). We will also implement the different edge operators such as Prewitt, Roberts, LoG, Canny and the results of these operators will be shown on various images.
\end{abstract}

\section{Introduction}

Image segmentation is the process of dividing an image into different regions such that each region is homogeneous. Image segmentation is the key behind image understanding. Image segmentation is considered as an important basic operation for meaningful analysis and interpretation of image acquired. It is a critical and essential component of an image analysis and/or pattern recognition system, and is one of the most difficult tasks in image processing, which determines the quality of the final segmentation. It is the prime area of research in computer vision. A number of image segmentation techniques are available, but there is no one single technique that is suitable to all the application. Researchers have extensively worked over this fundamental problem and proposed various methods for image segmentation. These methods can be broadly classified into seven groups: (1) Histogram thresholding, (2) Clustering (Fuzzy and Hard), (3) Region growing, region splitting and merging, (4) Discontinuity-based, (5) Physical model- based, (6) Fuzzy approaches, and (7) Neural network and GA (Genetic algorithm) based approaches. Discontinuitybased segmentation is one of the widely used techniques for monochrome image segmentation. In discontinuity-based approach, the partitions or subdivision of an image is based on some abrupt changes in the intensity level of images. Here, we mainly interest in identification of isolated points, lined and edges in an image.

Section II describes the related work in the area of edge detection algorithms. Section III discusses the image segmentation based on discontinuity-based approach. Under this approach, we analyses the point detection, line detection and edge detection techniques. A number of operator which are based on first-order derivatives and second-order derivatives such as Prewitt, Sobel, Roberts etc. are discussed in detail. Section IV performs the experimental results and discussion based on these operators. Finally, section $\mathrm{V}$ concludes the papers by analyzing and comparing the results of these operators.

\section{Related work}

A. Rosenfeld [1] develop a method for detecting edges between regions of different average gray level can be applied to detect a wide variety of "texture edges" in which two regions differ with respect to the average value of some local property. E. Adward [2] used a convolution method to find the efficient edge detector with an adjustable parameter $\mathrm{k}$ that may be selected to match the average interval between edges expected in the data. The Gaussian function $(\mathrm{G})$ has only one discontinuity and therefore generates less noise than the other convolving function. R. M. Haralick [3] uses the facet model to accomplish step edge detection. The essence of the facet model is that any analysis made on the basis of the pixel values in some neighborhood has its final authoritative interpretation relative to the underlying gray tone intensity surface of which the neighborhood pixel values are observed noise samples. He define an edge to occur in a pixel if and only if there is some point in the pixel's area having a negatively sloped zero crossing of the second directional derivative taken in the direction of a nonzero gradient at the pixel's center. J. Canny [4] describes a computational approach to edge detection. He defines detection and localization criteria for a class of edges, and present 
mathematical forms for these criteria as functional on the operator impulse response. A third criterion is then added to ensure that the detector has only one response to a single edge. N. R. Pal and S. K. Pal [5] reviews and summarizes some image segmentation algorithms which are based on fuzzy set, neural networks, thresholding, edge detection, clustering relaxation and Markov Random Field (MRF). They also address the issue of quantitative evaluation of segmentation results. T. Kanungo et al. [6] outline a methodology for summarizing many operating curves into a few performance curves. Their methodology is adapted from the human psychophysics literature and is general to any detection algorithm. The central concept is to measure the effect of variables in terms of the equivalent effect of a critical signal variable, which in turn facilitates the determination of the breakdown point of the algorithm. They demonstrate the methodology by comparing the performance of two-line detection algorithms. W. Y. Ma et al. [7] develop a framework for detecting and integrating intensity/color and texture discontinuities as well as illusory boundaries in images. They propose an edge flow model for boundary detection and image segmentation. Salient features of this approach include (1) use of a predictive coding model for identifying and integrating the different types of image boundaries, (2) boundary detection based on flow field propagation, and (3) very few "free" parameters that control the segmentation. C. Hampton et al. [8] summarized the various image segmentation techniques based on discontinuitybased, region-based, thresholding and histogram. K. S. Fu et al. [9] summarized some image segmentation techniques. These techniques can be categorized into three classes, (1) characteristic features thresholding or clustering, (2) edge detection, and (3) region extraction. N. Senthikumaran et al. [10] survey the theory of edge detection for image segmentation using soft computing approach based on the Fuzzy logic, Genetic Algorithm and Neural Network. S. S. Al-amri et al. [11] presented methods for edge segmentation of satellite image: they used seven techniques for this category; Sobel operator technique, Prewitt technique, Kiresh technique, Roberts technique, Laplacian technique, Canny technique and Edge Maximization Technique (EMT) and they are compared with one another so as to choose the best technique for edge detection segment image. P. Thakare [12] discussed about some image segmentation techniques like edge based, region based and integrated techniques and explains in brief the edge based techniques and their evaluation. They also focuses on edge based techniques and their evaluation. W. Kang et al. [13] classify and discuss main image segmentation algorithms; introduce the evaluation of image segmentation systematically; evaluate and compare basic, practical segmentation algorithms after a large number of comparative experiments. R. Maini et al. [14] analyzed and did the visual comparison of the most commonly used Gradient and Laplacian based edge detection techniques for problems of inaccurate edge detection, missing true edges, producing thin or thick lines and problems due to noise etc. P. Zhou et al. [15] discuss the basic theory of edge detection; its method based on the traditional canny operator, and proposes an improved algorithm based on the eight neighborhood gradient magnitude to overcome the disadvantages of being sensitive to noise in the calculation of the traditional canny operator gradient. The experimental results prove that this improved method can effectively detect the edge of the image, and the continuity of the edge is strong, and positioning accuracy is high.

\section{Discontinuity-based segmentation}

In discontinuity-based approach, the partitions or sub-division of an image is based on some abrupt changes in the intensity level of images. Here, we mainly interest in identification of isolated points, lined and edges in an image. To identify these, we use 3 × 3 Mask operation.

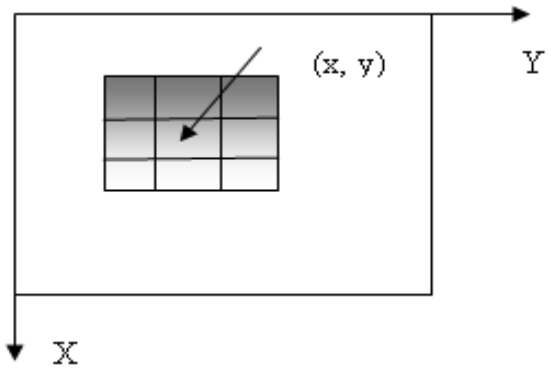

(a)

\begin{tabular}{|c|c|c|}
\hline $\mathrm{W}_{-1,-1}$ & $\mathrm{~W}_{-1,0}$ & $\mathrm{~W}_{-1,1}$ \\
\hline $\mathrm{W}_{0,-1}$ & $\mathrm{~W}_{0,0}$ & $\mathrm{~W}_{0,1}$ \\
\hline $\mathrm{W}_{1,-1}$ & $\mathrm{~W}_{1,0}$ & $\mathrm{~W}_{1,1}$ \\
\hline
\end{tabular}

(b)

Figure 1. (a) An image (b) 33 Mask

$$
R=\sum_{n=1}^{1} \sum_{n=1}^{1} w_{1,1} f(x+1, y+1)
$$


The discontinuity-based segmentation can be classified into three approaches: (1) Point detection, (2) Line detection, and (3) Edge detection.

\subsection{Point Detection [8] [16]}

A point is the most basic type of discontinuity in a digital image. The most common approach to finding discontinuities is to run an ( $\mathrm{x} n \mathrm{n})$ mask over each point in the image. The mask is as shown in figure 2.

\begin{tabular}{|c|c|c|}
\hline-1 & -1 & -1 \\
\hline-1 & 8 & -1 \\
\hline-1 & -1 & -1 \\
\hline
\end{tabular}

Figure 2. A mask for point detection

The point is detected at a location $(\mathrm{x}, \mathrm{y})$ in an image where the mask is centered. If the corresponding value of $\mathrm{R}$ such that

\section{$\|R\|>T$}

Where $\mathrm{R}$ is the response of the mask at any point in the image and $\mathrm{T}$ is non-negative threshold value. It means that isola ted point is detected at the corresponding value $(\mathrm{x}, \mathrm{y})$. This formulation serves to measures the weighted differences between the center point and its neighbors since the gray level of an isolated point will be very different from that of its neighbors [ ]. The result of point detection mask is as shown in figure 3 .

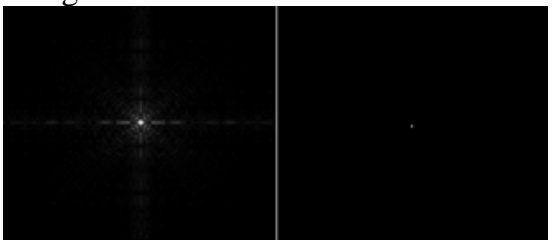

Figure 3. (a) Gray-scale image with a nearly invisible isolated black point (b) Image showing the detected point

\subsection{Line Detection [8] [16]}

Line detection is the next level of complexity in the direction of image discontinuity. For any point in the image, a response can be calculated that will show which direction the point of a line is most associated with. For line detection, we use two masks, $i^{\text {th }}$ and, $j^{\text {th }}$ mask. Then, we have

$$
R_{l}\|>\| R_{j} \|, \forall j \neq i
$$

It means that the corresponding points is more likely to be associated with a line in the direction of the mask $i$.

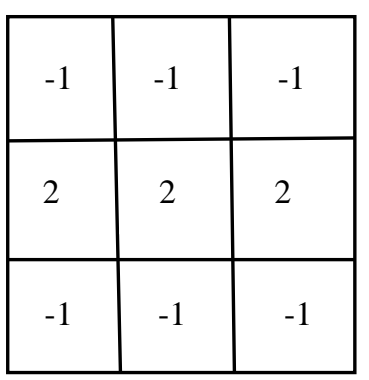

(a)

\begin{tabular}{|l|l|l|}
\hline-1 & 2 & -1 \\
\hline-1 & 2 & -1 \\
\hline-1 & 2 & -1 \\
\hline
\end{tabular}

(c)

\begin{tabular}{|c|c|c|}
\hline-1 & -1 & 2 \\
\hline-1 & 2 & -1 \\
\hline 2 & -1 & -1 \\
\hline
\end{tabular}

(b)

\begin{tabular}{|c|c|c|}
\hline 2 & -1 & -1 \\
\hline-1 & 2 & -1 \\
\hline-1 & -1 & 2 \\
\hline
\end{tabular}

(d)
Figure 4. Line Detector masks in (a) Horizontal direction (b) $45^{\circ}$ direction (c) Vertical direction

$$
\text { (d) }-45^{\circ} \text { direction }
$$

The greatest response calculation from these matrices will yield the direction of the given pixel []. The result of line detection mask is as shown in figure 5 .

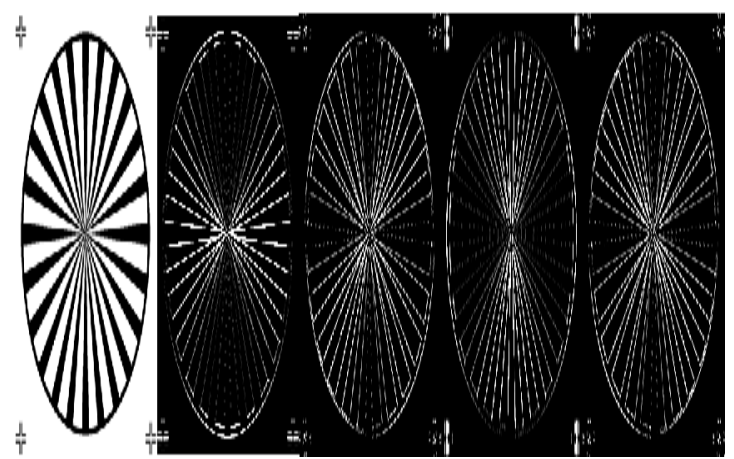

Figure 5. (a) Original Image (b) result showing with horizontal detector (c) with $45^{\circ}$ detector (d) with vertical detector (e) with $-45^{\circ}$ detector

With the help of lines detector masks, we can detect the lines in a specified direction. For example, we are interesting in finding all the lines that are one pixel thick, oriented at $-45^{\circ}$. For that, we take a digitized (binary portion of a wire-bond mask for an electronics circuit. The results are shown as in figure 6. 


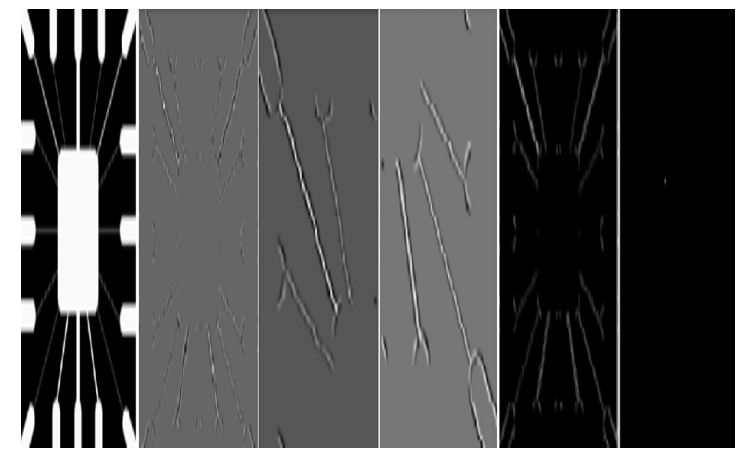

Figure 6. (a) Image of a wire-bond mask (b) Result of processing with the $-45^{\circ}$ detector (c) Zoomed view of the top, left region of $-45^{\circ}$ detector (d) Zoomed view of the bottom, right region of $-45^{\circ}$ detector (e) Absolute value of $-45^{\circ}$ detector (f) All points whose values satisfied the condition $g>=T$, where $g$ is the image in (e)

\subsection{Edge detection [8] [16]}

Since isolated points and lines of unitary pixel thickness are infrequent in most practical application, edge detection is the most common approach in gray level discontinuity segmentation. An edge is a boundary between two regions having distinct intensity level. It is very useful in detecting of discontinuity in an image. When the image changes from dark to white or vice-versa. The changes of intensity, first-order derivative and second-order derivative are shown in figure 7.

Dark White Dark White Dark White
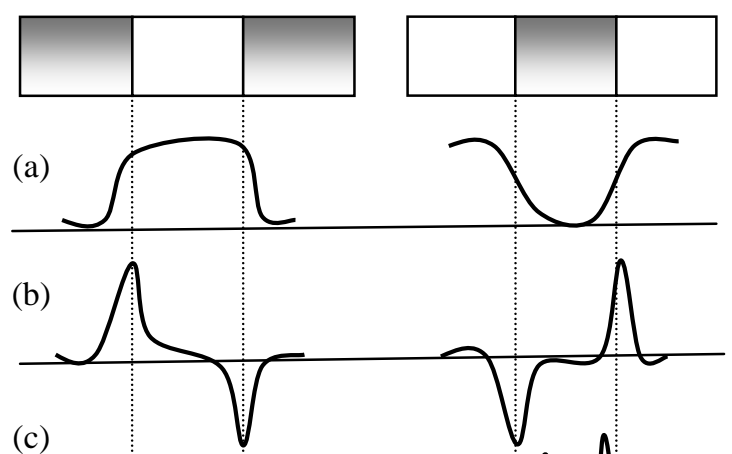

(c)

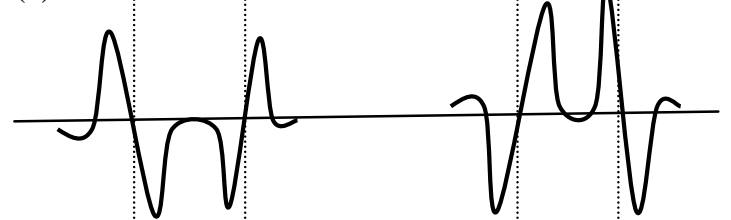

Figure 7. (a) Intensity profile (b) First-order derivatives (c) Second-order derivatives
3.3.1. First-order derivatives. First-order derivatives responds whenever there is discontinuity in intensity level. It is positive at the leading edge and negative at the trailing edge. Suppose we have an image $f(x, y)$ and gradient operator

$$
f=\left[\begin{array}{l}
G_{x} \\
G_{y}
\end{array}\right]=\left[\begin{array}{l}
\frac{g f}{\partial x} \\
\frac{g f}{\partial y}
\end{array}\right]
$$

Strength of $\mathbb{\nabla} f$ is given by

$$
\begin{aligned}
& \nabla f=\text { magnitudes of }(\overrightarrow{\nabla f}) \\
& =\sqrt{G_{x}^{2}+G_{y}^{2}} \\
& \mathbf{\|}\left\|G_{x}\right\|+\left\|G_{y}\right\|
\end{aligned}
$$

It gives the strength of edge at location $(\mathrm{x}, \mathrm{y})$ Direction of $\vec{\nabla} f$ is given by

$$
\alpha(x, y)=\tan ^{-1}\left(\frac{G_{x}}{G_{y}}\right)
$$

Where $\alpha(x, y)$ gives the direction of $\vec{\nabla} f$.

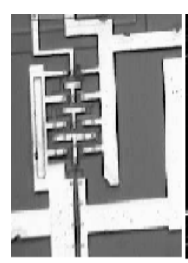

(a)

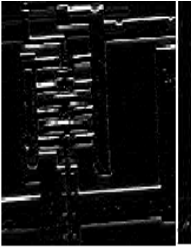

(b)

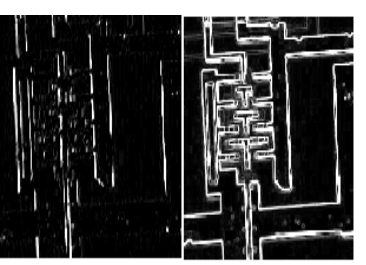

(c) (d)
Figure 8. (a) Original Image (b) $\left\|G_{x}\right\|$ component of the gradient along x-direction (c) $\left\|G_{y}\right\|$ component of the gradient along $y$-direction (d) Gradient Image $\left\|G_{x}\right\|+\left\|G_{y}\right\|$

There is several ways to calculate the image gradient:

\subsubsection{Prewitt Edge operator}

\begin{tabular}{|c|c|c|}
\hline-1 & -1 & -1 \\
\hline 0 & 0 & 0 \\
\hline-1 & -1 & -1 \\
\hline
\end{tabular}

\begin{tabular}{|l|l|l|}
\hline-1 & 0 & -1 \\
\hline-1 & 0 & -1 \\
\hline-1 & 0 & -1 \\
\hline
\end{tabular}

Figure 9. Masks used for Prewitt Edge operator

The mask finds the horizontal edges is equivalent to gradient in the vertical direction and the mask compute the vertical edges is equivalent to gradient in the horizontal direction. Using these two masks passing to the intensity image, we can find out $\mathrm{G}_{\mathrm{X}}$ and $\mathbf{G}_{y}$ component at different location in an image. So, 
we can find out the strength and direction of edge at that particular location (x, y).

\subsubsection{Sobel Edge operator}

\begin{tabular}{|c|c|c|}
\hline-1 & -2 & -1 \\
\hline 0 & 0 & 0 \\
\hline 1 & 2 & 1 \\
\hline
\end{tabular}

\begin{tabular}{|c|c|c|}
\hline-1 & 0 & 1 \\
\hline-2 & 0 & 2 \\
\hline-1 & 0 & 1 \\
\hline
\end{tabular}

Figure 10. Masks used for Sobel Edge operator

It gives the averaging effect over an image. It considers the effect due to the spurious noise in the image. It is preferable over prewitt edge operator because it gives the smoothing effect and by which we can reduce spurious edge which are generated because of noise present in the image.

\subsubsection{Second-order derivatives}

It is positive at the darker side and negative at the white side. It is very sensitive to noise present in an image. That's why it is not used for edge detection. But, it is very useful for extracting some secondary information i.e. we can find out whether the point lies on the darker side or the white side.

Zero-crossing: It is useful to identify the exact location of the edge where there is gradual transition of intensity from dark to bright region and vice-versa. There are several second-order derivative operators:

3.3.2.1. Laplacian operator. The Laplacian mask is given by:

\begin{tabular}{|c|c|c|}
\hline 0 & -1 & 0 \\
\hline-1 & 4 & -1 \\
\hline 0 & -1 & 0 \\
\hline
\end{tabular}

Figure 11. Masks used for Laplacian operator

$$
\nabla^{2}(f)=\frac{\partial^{2} f}{\partial x^{2}}+\frac{\partial^{2} f}{\partial y^{2}}
$$

If we consider the diagonal elements:

\begin{tabular}{|c|c|c|}
\hline-1 & -1 & -1 \\
\hline-1 & 8 & -1 \\
\hline-1 & -1 & -1 \\
\hline
\end{tabular}

\begin{tabular}{|l|l|l|}
\hline 1 & 1 & 1 \\
\hline 1 & 8 & 1 \\
\hline 1 & 1 & 1 \\
\hline
\end{tabular}

Figure 12. Masks used for Laplacian operator using 8-connectivity

It is not used for edge detection because it is very sensitive to noise and also leads to double edge. But, it is very useful for extracting secondary information. To reduce the effect of noise, first image will be smooth using the Gaussian operator and then it is operated by Laplacian operator. These two operations together is called LoG (Laplacian of Gaussian) operator.

\subsubsection{LoG operator}

The LoG mask is given by

\begin{tabular}{|c|c|c|c|c|}
\hline 0 & 0 & -1 & 0 & 0 \\
\hline 0 & -1 & 2 & -1 & 0 \\
\hline-1 & -2 & 1 & -2 & -1 \\
\hline 0 & -1 & -2 & -1 & 0 \\
\hline 0 & 0 & -1 & 0 & 0 \\
\hline
\end{tabular}

Figure 13. Masks used for LoG operator

The Gaussian operator is given by:

$$
\begin{aligned}
& h(x, y)=\exp \left(-\frac{x^{2}+y^{2}}{2 \sigma^{2}}\right) \quad(8) \\
& \text { where } x^{2}+y^{2}=r^{2} \\
& r^{2} h=\left(\frac{r^{2}-\sigma^{2}}{\sigma^{4}}\right) \exp \left(-\frac{r^{2}}{2 \sigma^{2}}\right)
\end{aligned}
$$

\subsubsection{Canny operator [4]}

It is very important method to find edges by isolating noise from the image before find edges of images, without affecting the features of the edges in the image and then applying the tendency to find the edges in the image and the critical value for threshold. 
i. Convolve image $f(r, c)$ with a Gaussian function to get smooth image $g(r, c)$.

$\mathrm{f}(\mathrm{r}, \mathrm{c})=\mathrm{f}(\mathrm{r}, \mathrm{c}) * \mathrm{G}(\mathrm{r}, \mathrm{c})$

ii. Apply first difference gradient operator to compute edge strength.

iii. Apply non-maximal or critical suppression to the gradient magnitude.

iv. Apply threshold to the non-maximal suppression image.

\section{Experimental results and discussion}

The various edge detectors are implemented in MATLAB using Image Processing Toolbox (IPT) and the result are shown as in figure 15 .

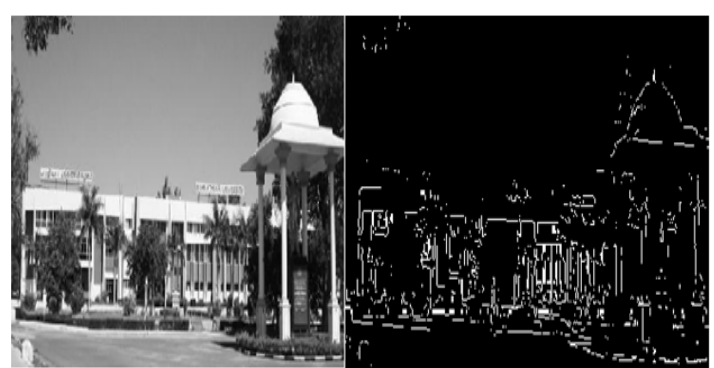

(a)

(b)

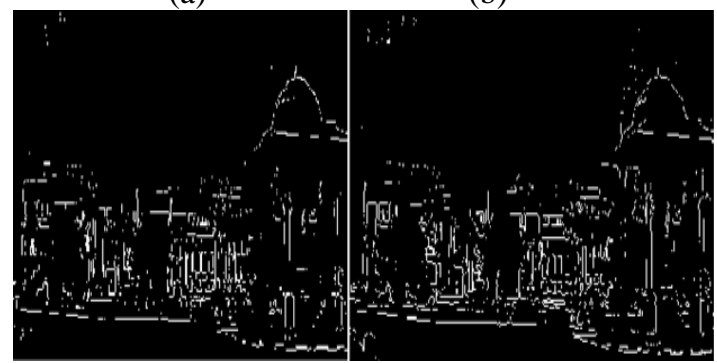

(c)

(d)

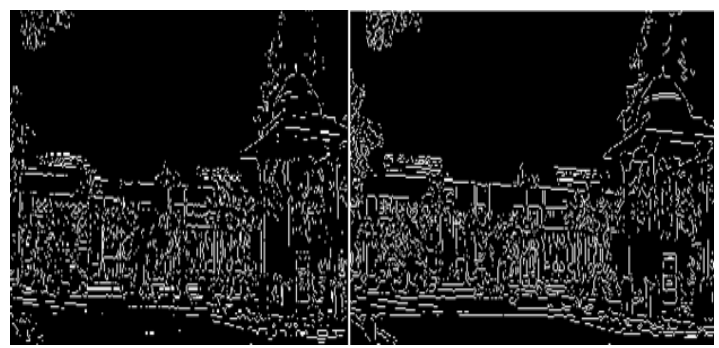

(e)

(f)

Figure 14. (a) Original image (b) Result using with Prewitt operator (c) Result using with Roberts operator (d) Result using with Sobel operator (e) Result using with LoG operator (f) Result using with Canny operator

These different operators respond to changes in gray level or average gray level. The gradient operators, not only respond to edges but also to isolates points. For Prewitt's operator the response to the diagonal edge is weak, while for Sobel's operator it is not that weak as it gives greater weights to points lying close to the point $(\mathrm{x}, \mathrm{y})$ under consideration. However, both Prewitt's and Sobel's operators posses greater noise immunity. The preceding operators are called the first difference operator. Laplacian, on the other hand, is a second difference operator. A good edge detector should be a filter with the following two features. First, it should be a differential operator, taking either a first or second spatial derivative of the image. Second, it should be capable of being tuned to act at any desired scale, so that large filters can be used to detect sharply focused fine details. The second requirement is very useful as intensity changes occur at different scales in an image. According to N. R. Pal [5], the most satisfactory operator fulfilling these conditions is the Laplacian of Gaussian (LoG) operator. The Gaussian part of the LoG operator blurs the image, wiping out all structures at scales much smaller than the $a$ of the Gaussian. The Gaussian blurring function is preferred over others because it has the desirable property of being smooth and localized in both spatial and frequency domains. According to canny [4], a good edge detector should have the following three properties: (1) low probability of wrongly marking non-edge points and low probability of failing to mark real edge points (i.e. good detection); (2) points marked as edges should be as close as possible to the centre of true edges (i.e. good localization); and (3) one and only one response to a single edge points (single response). Good detection can be achieved by maximizing signal to noise ratio (SNR), while for good localization; Canny used the reciprocal of an estimate of the r.m.s. distance of the marked edge from the center of the true edge. To maximize simultaneously both good detection and localization criteria, he maximized the product of SNR and the reciprocal of standard deviation (approximate) of the displacement of edge points. The maximization of the product is done subject to a constraint which eliminates multiple responses to a single edge points.

\section{Conclusion}

In this paper, we analysis various image segmentation techniques based on the discontinuitybased approach. In point-detection method, the point is detected at a location ( $\mathrm{x}, \mathrm{y})$ in an image where the mask is centered. In line detection method, we have two masks so that the corresponding points are more likely to be associated with a line in the direction of the one mask as compare to the one. In edge detection method, we analyses various operators such as Prewitt, Roberts, Sobel, LoG and canny. These 
different edge operators are implemented in matlab using image processing toolbox (IPT). The Prewitt's operator gives the weak response to the diagonal edge while Sobel operator gives the strong response. However, both these operators posses greater noise immunity. Laplacian of Gaussian (LoG) operator gives much better result as compare to Laplacian and Gaussian operator. In LoG, intensity changes occur at different scales in an image. The canny edge detector gives the better results as compare to other edge detectors because it provide good detection, good localization and single response.

\section{References}

[1] A. Rosenfeld, "A Nonlinear Edge Detection Techniques”, Proceedings of the IEEE, May 1970

[2] E. Argyle, “Techniques for edge detection”, Proc. IEEE, vol. 59, pp. 285-286, 1971.

[3] R. M. Haralick, “Digital Step Edges from Zero Crossing of Second Directional Derivatives", IEEE Transactions on Pattern Analysis and Machine Intelligence, vol. Pami-6, no. 1, Jan. 1986.

[4] J. F. Canny, "A computational approach to edge detection”, IEEE Trans. Pattern Anal. Machine Intell., vol. PAMI-8, no. 6, pp. 679-697, 1986.

[5] N. R. Pal and S. K. Pal, "A Review on Image Segmentation Techniques”, Pattern Recognition, vol. 26, no. 9, pp. 1277-1294, 1993.

[6] T. Kanungo, "A Methodology for Quantitative Performance Evaluation of Detection Algorithms”, IEEE Transactions on Image Processing, vol. 4, no. 12, Dec. 1995.

[7] W. Y. Ma and B. S. Manjunath, "Edge Flow: A Framework of Boundary Detection and Image Segmentation”, IEEE, 1997. http://www.vivaldi.ece.ussb.edu.

[8] C. Hampton, T. Persons, C. Wyatt and Y. Zhang, "Survey of Image Segmentation”, April 23, 1998.

[9] K. S. Fu, “A survey on image segmentation”, Pattern Recognition, vol. 13, pp. 3-16, 1981.

[10] N. Senthikumaran and R. Rajesh, "Edge detection techniques for image segmentation - A survey”, Proceedings for the International Conference on Managing Next Generation Software Applications (MNGSA-08), 2008, pp. 749-760.

[11] S. S. Al-amri, N. V. Kalyankar and S. D. Khamitkar, "Image Segmentation by using Edge Detection", International Journal on Computer Science and Engineering (IJCSE), vol. 02, no. 03, pp. 804-807, 2010.
[12] Puman Thakare, "A study of image segmentation and edge detection techniques", International Journal of Computer Science and Engineering (IJCSE), vol. 3, no. 2, Feb 2011.

[13] W. Kang, Q. Yang and R. Liang, "The Comparative Research on Image Segmentation Algorithms”.

[14] R. Maini and H. Aggarwal, "study and Comparsion of various Image Edge Detection Techniques", International Journal of Image Processing (IJIP), vol. 3, issue 1.

[15] P. Zhou, W. Ye, Y. Xia, Q. Wang, "An Improved Canny Algorithm for Edge Detection”, Journal of Computational Information System 7:5 (2011) 1516-1523.

[16] R. Gonzalez and R. Woods. Digital Image Processing. Addision-Wesley Publishing Company, Reading, MA, 1993. 